\title{
Evidence for mental subdivision of space by infants: 3- to 4-month-olds spontaneously bisect a small-scale area into left and right categories
}

\author{
Paul C. Quinn
}

Published online: 30 March 2012

(C) Psychonomic Society, Inc. 2012

\begin{abstract}
Young infants have been shown to represent the left versus right spatial category relations of a target object and a vertical referent bar. In the present study, we examined whether infants would represent left versus right when the vertical bar was removed from the stimulus display. In Experiment 1, 3- to 4-month-olds who had been familiarized with stimuli depicting a diamond appearing in different locations to the left or right of the vertical midline displayed a mean novel category preference for a stimulus depicting the diamond on the opposite side of the midline. In Experiment 2, another group of 3- to 4-month-olds discriminated the withincategory position changes of the diamond as it appeared to the left or right of the vertical midline. The results indicate that young infants can mentally bisect small-scale space into left versus right categories.
\end{abstract}

Keywords Categorization · Cognitive development - Spatial cognition

To experience a spatially coherent world, human perceivers must develop the ability to form category representations for spatial relations (Newcombe \& Huttenlocher, 2000). Over the past 15 years, a literature has emerged indicating that preverbal infants can represent spatial relation categories (Casasola, 2008; Quinn, 2007). For example, 3- to 4-month-olds who have been presented with stimuli in which a target shape (e.g., a dot or diamond) appears in discriminably different positions above or below a referent shape (i.e., a horizontal bar) will generalize looking time responsiveness to a stimulus in which the target shape has

P. C. Quinn $(\bowtie)$

Department of Psychology, University of Delaware,

Newark, DE 19716, USA

e-mail: pquinn@udel.edu moved to a novel location in the familiarized relation, but will display preferential responsiveness to a stimulus in which the target shape has moved to a location depicting the contrasting relation (e.g., Quinn, 1994; Quinn, Cummins, Kase, Martin, \& Weissman, 1996). Likewise, infants of the same age who have been presented with stimuli depicting the target shape in distinct locations to the left or right of a vertical referent bar will generalize responsiveness to a stimulus depicting the shape in a novel location on the same side of the bar, but will display preferential responsiveness to a stimulus depicting the shape on the opposite side of the bar (Quinn, 2004a; see also Gava, Valenza, \& Turati, 2009, for corroborating evidence in newborns). Importantly, infant performance in these studies could not have been based on distance information, because the shape on the opposite side of the bar was positioned the same distance away from the familiar shape locations as the shape in the novel location on the same side of the bar. In addition, the infants were not simply encoding the arbitrary crossing of a shape from one to the other side of the bar, because when the bar was diagonal and the changeover from familiar to novel category involved a shape changing location from, for example, below-left to above-right of the bar, the infants did not respond differentially (Quinn, 2004a). Taken together, the results suggest that young infants can represent at least some spatial relations as categories (i.e., above, below, left, right).

At the same time that a literature has been emerging on how infants represent spatial relation categories, related work has been examining the coding of spatial location in young children (Huttenlocher, Newcombe, \& Sandberg, 1994; Ortmann \& Schutte, 2010; Simmering, Schutte, \& Spencer, 2008). This work has been aimed in part at understanding children's representation of midline and its impact on perception and memory. In Huttenlocher et al., for example, 16- to 24-month-olds and 4- to 6-year-olds were found to represent a set of hiding locations inside a sandbox as 
a category with a prototypic central location. By contrast, older participants, 10-year-olds, subdivided the sandbox at the center into left and right categories. However, the developmental change from representing a single bounded space with a prototypic center to subdividing a bounded space into halves may not be qualitative, given that even 4-year-olds organized a bounded space into two halves under some conditions (i.e., representing a dot in a smaller scale space consisting of a rectangle depicted on paper).

To our knowledge, participants younger than 3 to 4 years of age have not been tested for their ability to mentally subdivide a space into left and right halves. Such evidence, if it were available, would strengthen the arguments that (a) the ability to subdivide space into bounded regions is available early in development, and (b) the transition from representing a bounded space as a single category with a prototypic center to subdividing that space into left and right categories, is quantitative in nature. The present experiments were therefore undertaken to determine whether young infants, 3 to 4 months of age, would perform like children between 4 and 10 years of age, and spontaneously subdivide a space into left and right categories, if provided with an age-appropriate task.

\section{Experiment 1}

In Experiment 1, 3- to 4-month-olds were tested for their ability to bisect a visual space into left versus right categories. In particular, to investigate whether 3- to 4-month-olds could represent a category of "diamond to the left of vertical midline," one group of infants was familiarized with four exemplars, each depicting a diamond in a different position to the left of the vertical midline of the display. Panels A and B of Fig. 1 display the locations of the diamond as it appeared in the familiar exemplars. Half of the infants were presented with the diamond appearing in each of the four corner positions of an imaginary rectangle located in the top half of the area to the left of the vertical midline (Panel A of Fig. 1). Immediately following familiarization, test trials were administered in which a novel exemplar from the familiar category (e.g., diamond in a new position to the left of the vertical midline) was paired with a novel exemplar from a novel category (e.g., diamond to the right of the vertical midline). It should be mentioned that the diamond in both test exemplars was moved the same distance from the average location of the diamond during familiarization (i.e., center of the imaginary rectangle). In the case of the novel exemplar from the familiar category, the diamond was moved below the rectangle's center. For the novel exemplar from the novel category, the diamond was moved the same distance right (from the rectangle's center) and consequently to the right of the vertical midline.
If infants can form a category representation for the spatial relation "diamond left of vertical midline," then they should display a preference for the novel exemplar from the novel category (i.e., diamond to the right of the vertical midline). The other half of the infants who were tested for formation of the category representation "diamond left of vertical midline" were familiarized with exemplars in which the diamond appeared in the four corner locations of an imaginary rectangle positioned in the bottom half of the area to the left of the vertical midline (Panel B of Fig. 1). Test trials followed in which a novel instance from the familiar category (diamond above the rectangle's center) was paired with a novel instance from a novel category (diamond to the right of the rectangle's center). Panels $\mathrm{C}$ and D of Fig. 1 display comparable experimental sequences designed to investigate formation of the category representation "diamond right of vertical midline" with a second group of infants. This experimental design matches with that of Quinn's (2004a) investigation of infant representation of left versus right categories, except that in the earlier study, infants could represent the depicted shape (i.e., the diamond) in relation to an externally available referent (i.e., vertical bar), whereas in the present study, the vertical bar was not present.

\section{Method}

Participants Participants were 24 healthy, full-term 3- to 4-month-olds (10 females) with mean age $=109.67$ days, $S D=10.34$ days. Two additional infants were tested, but did not complete the procedure because of fussiness. Participants in both experiments were predominantly Caucasian and from middle-class backgrounds.

Stimuli Stimuli were adapted from those used by Quinn (2004a). Each was created by arranging a black diamond onto a $17.7 \mathrm{~cm} \times 17.7 \mathrm{~cm}$ white posterboard card. The diamond was $0.9 \mathrm{~cm}\left(1.7^{\circ}\right)$ per side. The familiar element locations are depicted on the left side of Fig. 1. Center-to-center distance between the top and bottom elements of each rectangle was $5.0 \mathrm{~cm}\left(9.3^{\circ}\right)$, whereas distance between the left- and right-side elements was $3.5 \mathrm{~cm}\left(6.5^{\circ}\right)$. Center-to-center distance between the diamonds closest to the vertical midline and the midline itself was $2.0 \mathrm{~cm}\left(3.8^{\circ}\right)$. Location of the novel stimulus on the same side of the vertical midline (i.e., novel exemplar from the familiar category) was $6.5 \mathrm{~cm}\left(12.0^{\circ}\right)$ below or above the average of the familiar diamond locations (e.g., center of the imaginary rectangle); location of the novel stimulus on the opposite side of the vertical midline (i.e., the novel category exemplar) was $6.5 \mathrm{~cm}\left(12.0^{\circ}\right)$ to the right or left of the rectangle's center (shown on the far right side of Fig. 1).

Apparatus All infants were tested in a visual preference apparatus. The apparatus was a large, three-sided gray 
Fig. 1 Familiarization stimuli (a composite of the four exemplars) and test stimuli used to determine whether infants form category representations for left (panels A and B) or right (panels $\mathrm{C}$ and $\mathrm{D}$ ) of the vertical midline in Experiment 1

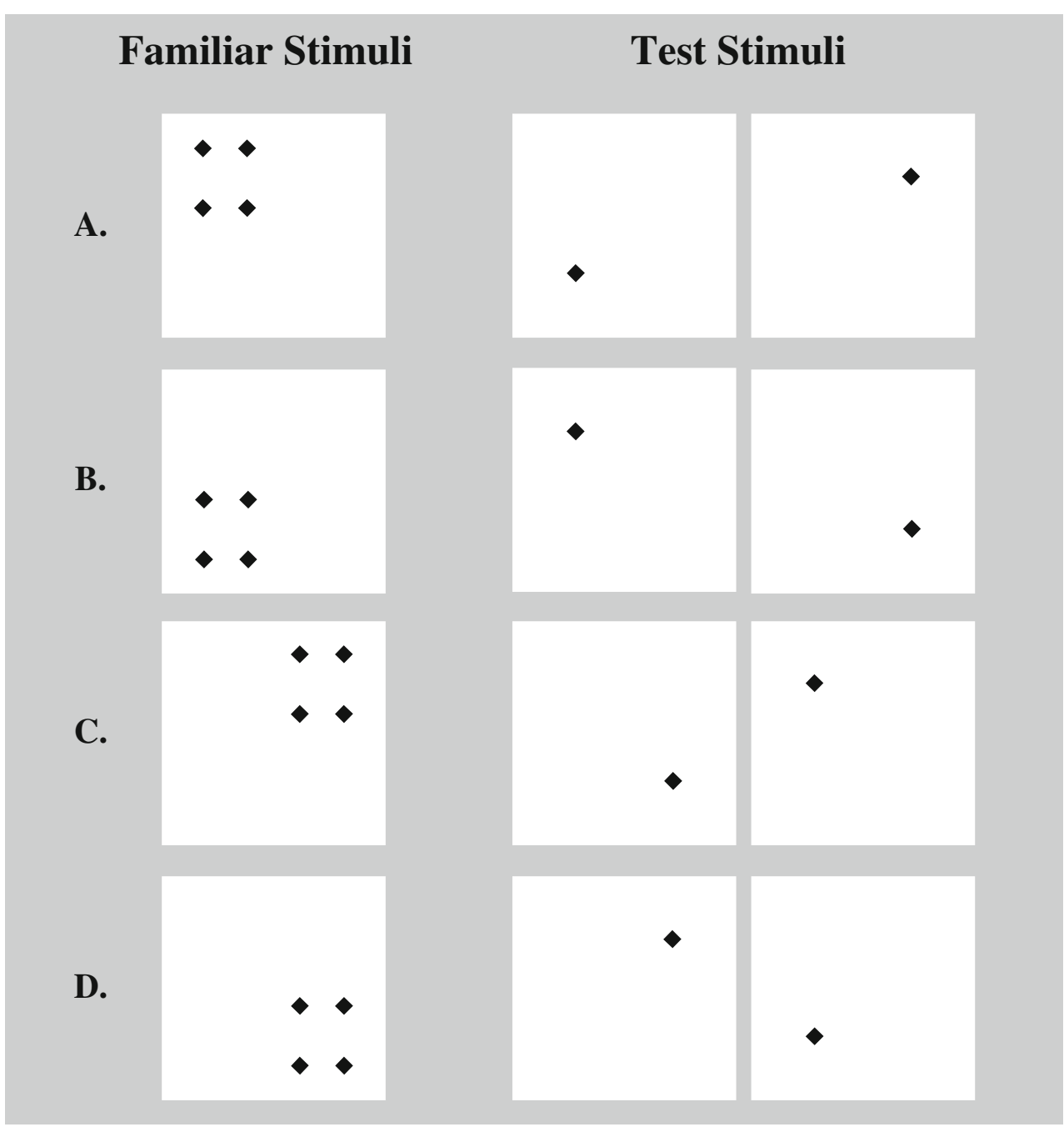

viewing chamber that was on wheels. It had a hinged, gray display panel onto which were attached two compartments to hold the stimuli. The stimuli were illuminated by a fluorescent lamp that was shielded from the infant's view. Center-to-center distance between compartments was $30.5 \mathrm{~cm}$, and on all trials, the display panel was situated approximately $30.5 \mathrm{~cm}$ in front of the infant. A $0.6-\mathrm{cm}$ peephole located midway between the compartments permitted an observer to record the infant's visual fixations. A second peephole, $0.9 \mathrm{~cm}$ in diameter, was located directly below the first peephole and permitted a Pro Video (Amityville, NY) CVC-120PH pinhole camera and Magnavox (Funai Corp., Torrance, CA) DVD recorder to record infants' gaze duration.

Procedure All infants were brought to the laboratory by a parent and were seated in a reclining position on the parent's lap. There were two experimenters who were both naive to the hypotheses under investigation. The first experimenter positioned the apparatus so that the midline of the infant's head was aligned with the midline of the display panel. The first experimenter also selected the appropriate stimuli, loaded them into the compartments of the display, and closed the panel, thereby exposing the stimuli to the infant. The parent was unable to see the stimuli.

During each trial, the first experimenter observed the infant through the small peephole and recorded visual fixations to the left and right stimuli by means of two $605 \mathrm{XE}$ Accusplit (San Jose, CA) electronic stop watches, one of which was held in each hand. The second experimenter timed the fixed duration of the trials and signaled the end of a trial. Between trials, the first experimenter opened the panel, changed stimuli, recorded infant looking times, and reclosed the panel. The first and second experimenters changed places for the test trials. The experimenter who presented the stimuli and measured infant fixations during familiarization now measured trial duration and signaled the end of each test trial, whereas the second experimenter presented the test stimuli and measured infant fixations. The second experimenter was always naive with respect to the familiar category in Experiment 1 or the familiar stimulus in Experiment 2. The two experimenters changed roles across infants. 
Interobserver agreement, as determined by comparing looking times measured by the experimenter using the center peephole, and an additional naive observer measuring looking times offline from DVD recordings, was calculated for the preference test trials of 12 infants participating across the two experiments. Average level of agreement was $98.01 \%(S D=1.04)$.

Twelve infants were randomly assigned to each of two groups, defined by the familiar category, left or right. All infants in the left group were administered four 15-s familiarization trials. On each trial, these infants were presented with two identical copies of a pattern in which a diamond appeared to the left of the vertical midline. For half of the infants in the left group, the diamond appeared, over the course of the four trials, in each of the four corners of an imaginary rectangle located in the top left quadrant of the stimulus (Panel A, Fig. 1). The order of presentation of the four diamond positions was randomized for each infant over the course of the four trials. Immediately after familiarization, two 10-s test trials were administered in which a novel stimulus from the familiar left category was paired with a novel stimulus from the novel right category. For the familiar category stimulus, the diamond appeared in a location below the center of the imaginary rectangle. For the novel category stimulus, the diamond was moved the same distance, but to a location to the right of the rectangle's center. Left-right positions of the familiar and novel category stimuli were counterbalanced across infants on the first test trial and were reversed on the second test trial. For the other half of the infants in the left group, the familiarization trials had the diamond appearing in the four corner locations of an imaginary rectangle positioned in the bottom left quadrant of the stimulus (Panel B, Fig. 1). These infants were tested with a familiar category exemplar in which the diamond appeared above the rectangle's center and a novel category exemplar in which the diamond was located the same distance to the right of the rectangle's center. The right group testing conditions (Panels C and D, Fig. 1) were symmetric to those of the left group.
Results and discussion

Familiarization trials Individual looking times were summed over left and right copies of the stimulus on each trial and averaged across the first two trials and last two trials. Mean looking times are shown in Table 1. An ANOVA (Trial Block [1-2 vs. 3-4] × Familiarization Condition [left vs. right]) of the individual looking times did not reveal any reliable effects, $F(1,22)<2.62, p>.11$, in each instance. The null effect of trial block is consistent with similar null effects observed in prior studies of spatial categorization by infants (e.g., Quinn et al., 1996) and suggests that infant attention was maintained by the trial-to-trial variation in dot location, an interpretation that is in agreement with the within-category discrimination results to be discussed in Experiment 2. What is important to emphasize is that, given the novelty preference data to be discussed directly below, lack of evidence for habituation did not preclude encoding of left-right category information associated with dot position.

Preference test trials Each infant's looking time to the novel category stimulus was divided by the looking time to both test stimuli and converted to a percentage score. The data are reported in percentage scores rather than looking times because the duration of the test trials (i.e., $10 \mathrm{~s}$ ) is shorter than the duration of the fixation trials (i.e., $15 \mathrm{~s}$ ). Test trials were kept to a short duration to capture a presumed burst of differential responsiveness toward the novel versus familiar category stimulus. If test trials had been made longer, any initial advantage in responsiveness to the novel category stimulus could have conceivably begun to subside because that stimulus was now becoming familiar. Mean novel category preference scores for the left and right familiarization conditions are shown in Table 1 . Both means were reliably different from the chance value of $50 \%$, and the two were not different from each other, $t(22)=0.24, p>.20$, two-tailed. The results indicate that the infants could form distinct category representations for the left versus right relations of a diamond and the vertical midline of the stimulus display.

Table 1 Mean fixation times (in seconds) during the familiarization trials, and mean novel category preference scores (in percentages) during the test trials of Experiment 1

\begin{tabular}{|c|c|c|c|c|c|c|c|c|}
\hline \multirow[b]{3}{*}{ Familiarization Category } & \multirow[b]{3}{*}{$N$} & \multicolumn{4}{|c|}{ Fixation Time } & & & \\
\hline & & \multicolumn{2}{|c|}{ Trials $1-2$} & \multicolumn{2}{|c|}{ Trials $3-4$} & \multicolumn{3}{|c|}{ Novelty Preference } \\
\hline & & $M$ & $S D$ & $M$ & $S D$ & $M$ & $S D$ & $t^{\mathrm{a}}$ \\
\hline Left & 12 & 6.18 & 2.85 & 5.57 & 1.99 & 64.11 & 13.77 & $3.55 * * *$ \\
\hline Right & 12 & 6.61 & 3.56 & 5.60 & 3.02 & 62.48 & 19.28 & $2.24 * *$ \\
\hline Combined & 24 & 6.39 & 3.16 & 5.59 & 2.50 & 63.29 & 16.40 & $3.97 * * * *$ \\
\hline
\end{tabular}

${ }^{\mathrm{a}} t$ tests compared mean preference scores with chance performance of $50 \%$. $* * p<.05$, two-tailed; $* * * p<.01$, two-tailed; $* * * *<.001$, two-tailed 


\section{Experiment 2}

Given that categorization is defined as an equivalent response to instances from the same class that are discriminably different, it is necessary to demonstrate that the instances of the familiarized category can be differentiated. Although within-category discrimination ability for exemplars of leftright relations was reported in Quinn (2004a), encoding of location of the exemplars in the earlier study could have been facilitated by the presence of the external bar. It therefore becomes important to demonstrate within-category discrimination for the exemplars used in the present study.

As in Quinn (2004a), the procedure used to measure discriminability involved presenting a randomly selected exemplar from within the left or right categories for a single 15-s trial. A single 15-s trial was used because it corresponded with the length of time each exemplar was presented during familiarization in the test of categorization in Experiment 1. Had four 15-s trials been used as in Experiment 1, it would have been possible for the infants to have had $60 \mathrm{~s}$ worth of encoding time with a given stimulus (as compared with a maximum of $15 \mathrm{~s}$ worth of encoding per stimulus in Experiment 1), and one could argue that Experiment 2 would be providing an overly optimistic estimate of how well infants discriminated the location changes of the diamond depicted in the stimuli used in Experiment 1. Immediately following familiarization, the familiar stimulus was paired with a novel exemplar from the same category, also randomly selected, for two 10 -s test trials.

\section{Method}

Participants Participants were 24 healthy, full-term 3- to 4month-olds ( 12 females) with mean age $=112.58$ days, $S D=$ 8.08 days. Two additional infants were tested but failed to complete the procedure due to fussiness.

Stimuli Stimuli were the same as those used in Experiment 1.

Procedure The within-category discrimination tests followed from the categorization tests of Experiment 1. In Experiment 1, each infant was familiarized with four exemplars, each depicting a diamond positioned in one of four corners of an imaginary rectangle located in one of the four quadrants of the stimulus. The novel instance of the familiar category shown during test trials contained a diamond shifted below or above the diamond locations shown during familiarization. In Experiment 2, each infant was tested for discrimination between two of the five exemplars used to represent the familiar category in a given familiarization and preference test condition in Experiment 1. The pair of exemplars was randomly chosen for each infant, as was which member of the pair would serve as the familiar stimulus. Familiarization consisted of a single 15-s trial, during which the familiar stimulus was shown in both compartments of the display stage. Immediately after familiarization, the familiar stimulus was paired with the novel stimulus for two 10 -s test trials. Left-right positioning of the novel stimulus was counterbalanced across infants on the first test trial and reversed on the second test trial. Half of the participants were tested with the diamond locations to the left of the vertical midline, and the other half were tested with diamond positions to the right of the vertical midline.

\section{Results and discussion}

Familiarization trial Individual looking times were summed over left and right copies of the stimulus and averaged across infants. Mean looking times to the stimuli presented to the left or right of the vertical midline are shown in Table 2. The difference was not significant, $t(22)=0.31, p>.20$, two-tailed.

Preference test trials Each infant's looking time to the novel stimulus was divided by the looking time to both test stimuli and converted to a percentage score. Mean preference scores for the novel stimulus for the left and right familiarization conditions are shown in Table 2. Both means were revealed by $t$ tests to be reliably above chance $(50 \%)$. In addition, the two means were not reliably different from each other, $t(22)=0.29, p>.20$, two-tailed. Infants who had been familiarized with a stimulus depicting the diamond to the left or right of the vertical midline preferred a stimulus depicting the diamond in a novel position on the same side of that midline. These results indicate that 3- to 4-month-olds were able to discriminate the exemplars from within the left and right categories, and that the novel category preference scores observed in Experiment 1 were unlikely to have arisen from within-category discrimination failure.

Table 2 Mean fixation times (in seconds) during the familiarization trial, and mean novelty preference scores (in percentages) during the preference test trials in Experiment 2

\begin{tabular}{lllllll}
\hline Familiarization & \multicolumn{2}{l}{ Familiarization Trial } & & \multicolumn{2}{l}{ Novelty Preference } \\
\cline { 2 - 3 } \cline { 6 - 8 } \cline { 5 - 7 } Stimulus & $M$ & $S D$ & & $M$ & $S D$ & $t^{\mathrm{a}}$ \\
\hline Left & 6.21 & 3.00 & & 60.54 & 17.14 & $2.13^{*}$ \\
Right & 6.61 & 3.41 & & 62.63 & 17.68 & $2.47^{* *}$ \\
Combined & 6.41 & 3.15 & & 61.59 & 17.06 & $3.33^{* * *}$ \\
\hline
\end{tabular}

${ }^{\mathrm{a}} t$ tests compared mean preference scores with chance performance of $50 \%$. ${ }^{*} p<.05$, one-tailed; ${ }^{* *} p<.05$, two-tailed; ${ }^{* * *} p<.01$, two-tailed 


\section{General discussion}

An earlier study of young infant categorization of spatial relation information provided evidence that infants could represent the left versus right relations between a diamond shape and a vertical bar (Quinn, 2004a). In the present study, we repeated the procedure of Quinn (2004a), but in this instance, the stimuli did not contain a vertical bar positioned in the vertical midline of the display. In Experiment 1, 3- to 4-month-olds who were familiarized with a diamond appearing in distinct locations to the left or right of the vertical midline displayed a novel category preference for an exemplar displaying the diamond on the opposite side of the midline. In Experiment 2, we showed that the preferences of Experiment 1 were not a result of failure to discriminate the exemplars from within the left and right categories. Taken together, the findings suggest that the infants were able to form category representations of the spatial relations left and right, and importantly, they were able to do so without the assistance of an external landmark that in the prior study came in the form of the vertical bar. To our knowledge, this is the first evidence that a developmental population as young as 3 to 4 months of age can organize a bounded space into subsections. The outcomes imply that the ability to mentally divide space into left and right regions that was previously observed in a small-scale paper task with 4-yearolds, and in a larger-scale sandbox task with 10-year-olds (Huttenlocher et al., 1994; see also Ortmann \& Schutte, 2010; Simmering et al., 2008), is operational from the earliest beginnings of development.

It is noteworthy that in the above-below version of the spatial categorization task in which infants formed category representations for the above versus below relations of a shape and a horizontal bar, the infants did not form the category representations for above versus below when the horizontal bar was removed from the stimuli (Quinn, 1994). This earlier finding stands in contrast to that reported presently in which infants in the same age range formed category representations for left versus right when the vertical bar was removed from the stimuli. The results are consistent with findings that left-right cues dominate above-below cues when both are available (Nicoletti, Umiltà, Tressoldi, \& Marzi, 1988), evidence that left-right symmetry is more salient than above-below symmetry (Corballis \& Beale, 1983), the outcome that categorical perception is sharper around vertical than it is around horizontal (Quinn, 2004b), and the idea that attention naturally distinguishes between left and right because of hemispheric specialization (Chatterjee, Southwood, \& Basilico, 1999).

The data on differences in the processing of direction away from the vertical versus horizontal axes in infants are also consistent with the developmental literature on the emergence of orientation perception where children have been shown to distinguish upright (i.e., vertical) from nonupright (i.e., all other orientations) categories initially, and are only subsequently observed to parse the nonupright category into diagonal, upside-down, and horizontal categories (Braine, 1978). Moreover, when asked to copy simple forms such as a "+," children are more likely to include an elongated vertical axis with two short horizontal axes extending from the vertical than they are to include an elongated horizontal axis with two short vertical axes extending from the horizontal (Tada \& Stiles, 1996). The data are further consistent with dissociations between processing above--below and left-right that have been reported in a variety of participant populations, including healthy adults and children (Clark, 1973; Logan, 1995), children with Williams syndrome (Landau, 2003), and developmental neuropsychological patients (McCloskey, Valtonen, \& Sherman, 2006). What the present findings add to the literature on developmental spatial cognition is that the ability to represent some spatial relations (i.e., left vs. right of a vertical axis) differentially from others (above vs. below of a horizontal axis) may be derived from the initial settings of our perceptual-cognitive system.

Author Note The present research was supported by NIH Grant HD-46526. The author thanks Matthew M. Doran and Laurie A. Yarzab for their assistance, and two anonymous reviewers for their comments on the initial submission.

\section{References}

Braine, L. G. (1978). A new slant on orientation perception. American Psychologist, 33, 10-20.

Casasola, M. (2008). The development of infants' spatial categories. Current Directions in Psychological Science, 17, 21-25.

Chatterjee, A., Southwood, M. H., \& Basilico, D. (1999). Verbs, events and spatial representations. Neuropsychologia, 37, $395-402$.

Clark, H. H. (1973). Space, time, semantics, and the child. In T. E. Moore (Ed.), Cognitive development and the acquisition of language (pp. 28-64). New York, NY: Academic Press.

Corballis, M. C., \& Beale, I. L. (1983). The ambivalent mind. Chicago, IL: Nelson-Hail.

Gava, L., Valenza, E., \& Turati, C. (2009). Newborns' perception of left-right spatial relations. Child Development, 80, 1797-1810.

Huttenlocher, J., Newcombe, N., \& Sandberg, E. H. (1994). The coding of spatial location in young children. Cognitive Psychology, 27, $115-147$.

Landau, B. (2003). Axes and direction in spatial language and spatial cognition. In E. van der Zee \& J. Slack (Eds.), Representing direction in language and space (pp. 18-38). Oxford, England: Oxford University Press.

Logan, G. D. (1995). Linguistic and conceptual control of visual spatial attention. Cognitive Psychology, 28, 103-174.

McCloskey, M., Valtonen, J., \& Sherman, J. C. (2006). Representing orientation: A coordinate-system hypothesis and evidence from developmental deficits. Cognitive Neuropsychology, 23, 680-713. 
Newcombe, N., \& Huttenlocher, J. (2000). Making space: The development of spatial representation and reasoning. Cambridge, MA: MIT Press.

Nicoletti, R., Umiltà, C., Tressoldi, E. P., \& Marzi, C. A. (1988). Why are left-right spatial codes easier to form than above-below ones? Perception \& Psychophysics, 43, 287-292.

Ortmann, M. R., \& Schutte, A. R. (2010). The relationship between the perception of axes of symmetry and spatial memory during early childhood. Journal of Experimental Child Psychology, 107, 368-376.

Quinn, P. C. (1994). The categorization of above and below spatial relations by young infants. Child Development, 65, 58-69.

Quinn, P. C. (2004a). Spatial representation by young infants: Categorization of spatial relations or sensitivity to a crossing primitive? Memory \& Cognition, 32, 852-861.

Quinn, P. C. (2004b). Visual perception of orientation is categorical near vertical and continuous near horizontal. Perception, 33, 897-906.
Quinn, P. C. (2007). On the infant's prelinguistic conception of spatial relations: Three developmental trends and their implications for spatial language learning. In J. M. Plumert \& J. P. Spencer (Eds.), The emerging spatial mind (pp. 117-141). New York, NY: Oxford University Press.

Quinn, P. C., Cummins, M., Kase, J., Martin, E., \& Weissman, S. (1996). Development of categorical representations for above and below spatial relations in 3- to 7-month-old infants. Developmental Psychology, 32, 642-650.

Simmering, V., Schutte, A. R., \& Spencer, J. P. (2008). Generalizing the dynamic field theory of spatial cognition across real and developmental time scales. Brain Research, 1202, 6886.

Tada, W. L., \& Stiles, J. (1996). Developmental change in children's analysis of spatial patterns. Developmental Psychology, 32, 951970 . 